\title{
Enhanced Efficiencies for High-Concentration, Multijunction PV Systems by Optimizing Grid Spacing under Nonuniform Illumination
}

\author{
Pratibha Sharma, Alex W. Walker, Jeffrey F. Wheeldon, \\ Karin Hinzer, and Henry Schriemer
}

SUNLAB, University of Ottawa, Ottawa, ON, Canada K1N 6N5

Correspondence should be addressed to Pratibha Sharma; psharm2@uottawa.ca

Received 7 December 2013; Accepted 1 June 2014; Published 7 August 2014

Academic Editor: Przemyslaw Rupnowski

Copyright (c) 2014 Pratibha Sharma et al. This is an open access article distributed under the Creative Commons Attribution License, which permits unrestricted use, distribution, and reproduction in any medium, provided the original work is properly cited.

\begin{abstract}
The design of a triple junction solar cell's front contact grid can significantly affect cell conversion efficiency under high concentration. We consider one aspect of grid design, choosing a linear grid within a distributed resistance cell model to optimize finger spacings at concentrations between 500 and 2500 suns under uniform and nonuniform illumination. Optimization for maximum efficiency under Gaussian irradiance profiles is done by SPICE analysis. Relative to the optimized uniform illumination designs, we find enhancements of $0.5 \%$ to $2 \%$ in absolute efficiencies for uniform spacing. Efficiency enhancement with nonuniform spacing under nonuniform illumination is also evaluated. Our model suggests that, at lower concentrations $(<1000$ suns), the penalty for using uniformly spaced fingers instead of nonuniformly spaced fingers is $<0.1 \%$. However, at a concentration of 2500 suns the penalty increases to $0.3 \%$. Thus, relative to a uniform irradiance optimization, an absolute efficiency increase of $2.3 \%$ can be attained for an optimized nonuniform spacing given the Gaussian irradiance profile under consideration.
\end{abstract}

\section{Introduction}

Multijunction solar cells based on III-V semiconductors are the most efficient of all photovoltaic technologies, with a record efficiency of $44.7 \%$ under concentration recently reported [1]. Not only does concentration permit the balance of concentrator photovoltaic system costs to offset the expensive material costs of multijunction solar cells, but it also enhances cell efficiency through the logarithmic increase in open circuit voltage [2]. The effects of concentration lead to a nonuniform profile that is not effectively flattened by secondary optic solutions [3]. Concentrator PV system optics often generate profiles that peak sharply at the center of the cell (Figure 1(a)) and follow a Gaussian distribution [4]. Peak-to-average irradiance ratios (PAR) typically range between 2 and 10 [2], but greater PARs are not unknown [5]. The nonuniformity in irradiance leads to a nonuniformity in current production that can decrease cell efficiency $[6,7]$.
The decrease in cell performance becomes increasingly evident at higher concentrations, where increasing current densities drive higher series resistance $\left(I^{2} R\right)$ losses. The impact of these $I^{2} R$ losses is a reduction in fill factor and therefore a reduction in conversion efficiency [8]. For uniform irradiance, a linear grid with even finger spacing, as shown in Figure 1(b), achieves maximum extraction efficiency regardless of such losses. This is not the case for nonuniform irradiance, where the consequent nonuniform current production within each subcell also leads to lateral currents that exacerbate carrier recombination. Continued reliance on uniform irradiance grid designs is therefore a cause for concern [6], motivating analyses of more realistic scenarios.

Simulation tools such as TCAD Sentaurus allow for valuable understanding of how solar cell performance depends on material properties and device structure [9]. However, as their complexity drives long computational times, usage of such tools is limited to simulating a small symmetrical 


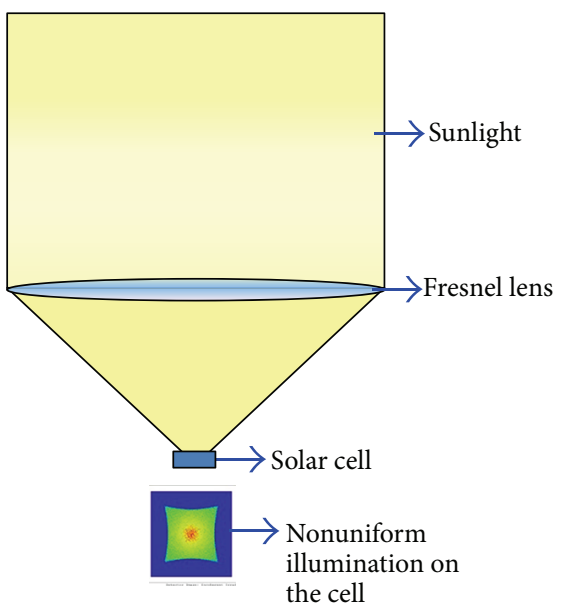

(a)

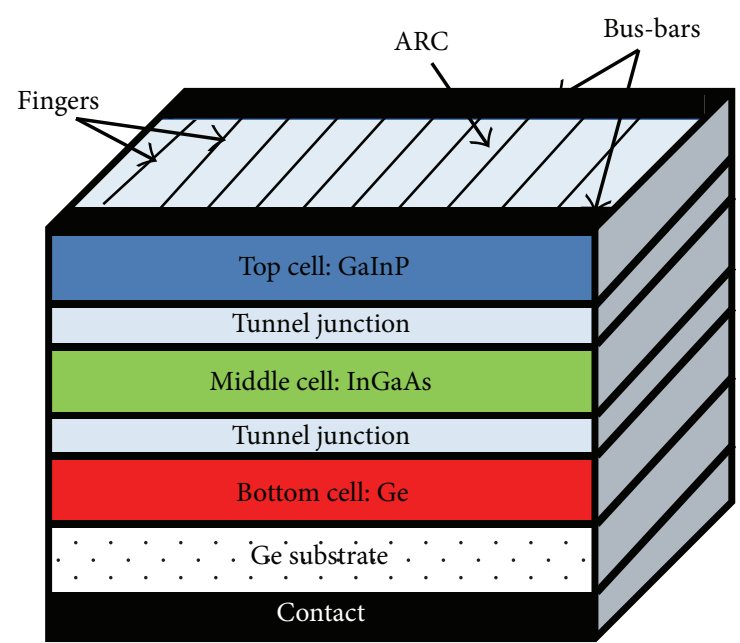

(b)

FIgURE 1: (a) A Fresnel-lens based CPV system showing nonuniform illumination on the solar cell. (b) Triple junction solar cell structure with a linear grid design; the bus-bars are outside the illumination area.

element of the device. Therefore, electrical models designed in SPICE (simulation program with integrated circuit emphasis) have been used to characterize the spatial dependence of solar cell performance. Due to their inability to simulate the effects of distributed resistance with varying irradiance profiles, classical lumped SPICE models do not permit realistic simulation. Hence, distributed resistance models, capable of simulating actual cell areas, are required for evaluating the effects of nonuniform illumination.

In this paper, we use a two-diode equivalent circuit for each subcell in a two-dimensional (2-D) distributed resistance model to address non-uniform current production under various Gaussian irradiance profiles. We report on the efficiency enhancements obtained by grid spacing optimization using realistic irradiance profiles at concentrations of 500,1000, 2000, and 2500 suns with both uniform and nonuniform grid spacing. The results are compared with those obtained from the corresponding uniform irradiance analyses.

\section{Numerical Model}

2.1. Device Structure and Parameter Extraction. In order to generate values for equivalent circuit parameters that act as an input to the SPICE model, $I-V$ curves for each subcell are required. These can be obtained either by using experimental data from isotypes or by simulating solar cell structures using TCAD tools $[9,10]$. In this work, we have designed a triple-junction solar cell (TJSC) structure using TCAD Sentaurus by Synopsys. The simulation has been performed on a symmetrical element of a lattice-matched TJSC with 5\% shading due to an ohmic contact on a highly doped GaAs cap layer. The structure consists of three subcells composed of GaInP, InGaAs, and Ge layers on a Ge substrate [9] connected in series using low-resistance tunnel junctions [11]. Previous variants of the model have been validated with experimental
TABLE 1: Triple junction solar cell parameters as obtained by curvefitting simulated subcell IV curves at 1 sun.

\begin{tabular}{lccc}
\hline Parameter & Top subcell & Middle subcell & Bottom subcell \\
\hline$I_{01}(\mathrm{~A})$ & $1.0 \times 10^{-25}$ & $1.6 \times 10^{-19}$ & $1.6 \times 10^{-6}$ \\
$I_{02}(\mathrm{~A})$ & $1.0 \times 10^{-20}$ & $6.5 \times 10^{-12}$ & $1.3 \times 10^{-5}$ \\
$I_{L}(\mathrm{~mA})$ & 12.9 & 12.9 & 21.5 \\
$R_{\mathrm{sh}}(\mathrm{k} \Omega)$ & 10 & 10 & 3.5 \\
\hline
\end{tabular}

results. Since a two-diode model yields more accurate results than a one-diode model [12], solar cell parameters have been determined for each subcell by curve-fitting the subcell $I-V$ curves to

$$
I=I_{L}-I_{D 1}-I_{D 2}-\frac{\left(V+I R_{S}\right)}{R_{\mathrm{sh}}},
$$

where the individual diode equations are

$$
\begin{aligned}
& I_{D 1}=I_{01}\left[\exp \left(\frac{q\left(V+I R_{S}\right)}{k T}\right)-1\right], \\
& I_{D 2}=I_{02}\left[\exp \left(\frac{q\left(V+I R_{S}\right)}{2 k T}\right)-1\right] .
\end{aligned}
$$

$I_{L}$ is the light-generated current (which is directly proportional to the solar irradiation), $I_{01}$ and $I_{02}$ are reverse saturation currents, $q$ is the electronic charge, $k$ is the Boltzmann constant, $R_{S}$ is the series resistance, $R_{\mathrm{sh}}$ is the shunt resistance, $V$ is the output voltage, and $I$ is the output current of the solar cell.

Since the values of $I, V$, and $R_{S}$ are known from the subcell $I-V$ curves and from the sheet resistances of the TJSC structure, the remaining unknowns, $I_{L}, I_{01}, I_{02}$, and $R_{\text {sh }}$, can be determined by curve-fitting using the least-squares method [13]. The parameters thus obtained are shown in Table 1 and have errors of less than $2 \%$. 


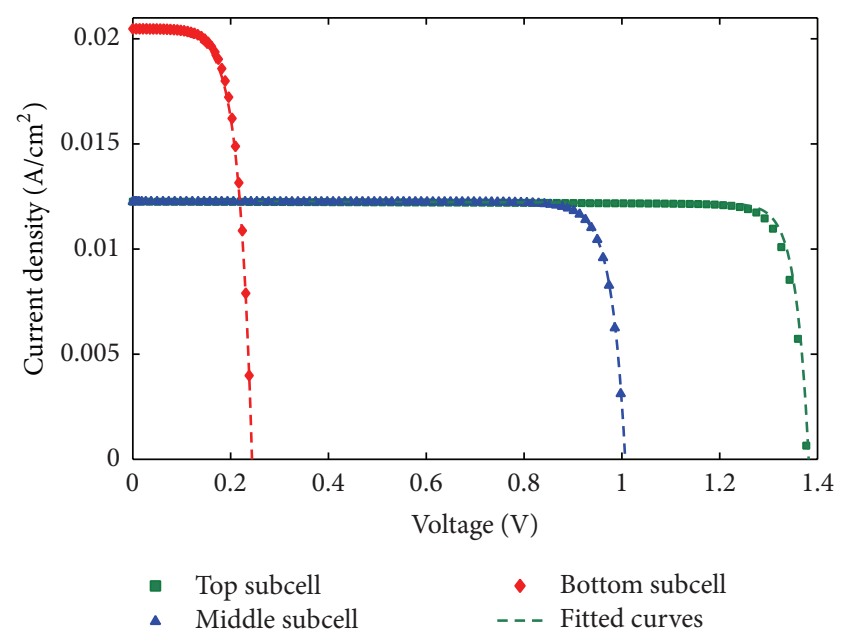

FIgURE 2: Simulated subcell $J-V$ data for the TJSC obtained using TCAD Sentaurus (at $300 \mathrm{~K}$ and 1 sun) along with the least-squares, curve-fitted data.

Figure 2 shows the fitted subcell current-density versus voltage $(J-V)$ curves along with the simulated $(J-V)$ curves obtained for this structure using TCAD Sentaurus.

\subsection{2-Dimensional Distributed Resistance Model. Equivalent} circuit representation of solar cells is useful for CPV system analysis [14-17]. In this work, a 2-D distributed resistance model for a TJSC is used [13]. This model, designed using LTSPICE, is an improvement over the 2-D model described by Nishioka et al. [14] as it includes equivalent circuits for the directly illuminated regions and for the shaded areas underneath the metal contact. The inclusion of equivalent circuits for the shaded areas is essential to analyze the effects of nonuniform illumination on cell efficiency [16].

Figure 3 presents a detailed circuit diagram for the SPICE model depicting illuminated and shaded equivalent circuits that comprise the repeated functional block. Since finger spacing is variable (given constant finger width), the number of functional blocks $n$ varies with spacing for each simulation and is given by

$$
n=\frac{A_{t}}{S_{f}+W_{f}},
$$

where $A_{t}$ is the total cell area, $S_{f}$ is the finger spacing, and $W_{f}$ is the finger width. Narrower spacing requires a larger number of functional blocks to represent the physical extent of the TJSC being modeled.

The linear grid means a 2-D model will suffice to describe the TJSC. The model incorporates lateral resistance components connecting the functional blocks across the rows $\left(R_{\text {lat }}\right)$, tunnel junction resistances $\left(R_{T}\right)$ connecting the subcells in series, and contact resistances $\left(R_{\mathrm{se}}\right)$ for the electrodes. The number of function blocks for a fixed cell dimension determines the lateral resistance values $[13,18]$. Electrode resistances are assumed to be negligible, finger widths are fixed at $5 \mu \mathrm{m}$, and the active cell area is equal to $1 \mathrm{~cm}^{2}$. The model therefore accounts for shading losses due to the fingers

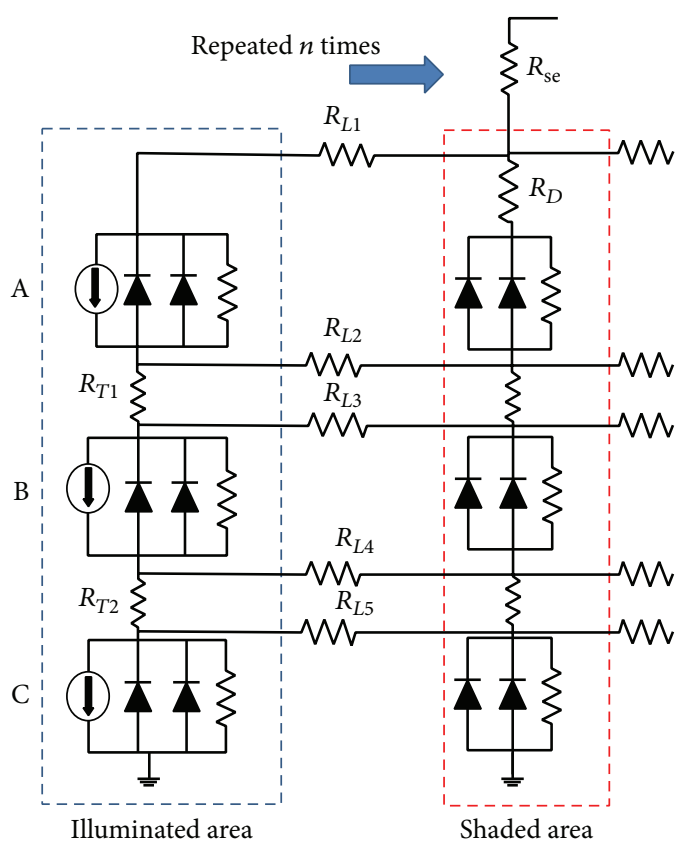

FIGURE 3: 2-D distributed resistance model developed in SPICE showing the two-diode equivalent circuits for each of the subcells where (A) top subcell, (B) middle subcell, and (C) bottom subcell are connected in series using tunnel junctions. An illuminated and a shaded unit form a complete functional block that is repeated $n$ times.

and ohmic losses due to series resistances. Bus bars are assumed to be outside the active cell area and are not included in the model. Shading losses and series resistances due to bus bars are neglected for our simulations.

\section{Optimization}

In order to optimize finger spacing, the solar cell conversion efficiency is assumed to be a unimodal objective function with one maximum. A derivative-free optimization approach is adopted to maximize cell efficiency. In order to reduce the number of iterations and for a faster convergence, a Goldensection search algorithm is used. Finger spacings range from $50 \mu \mathrm{m}$ to $300 \mu \mathrm{m}$, for a shading range of $\sim 2 \%$ to $10 \%$, which is typical for solar cell designs. Convergence of the optimization process is ensured by requiring the absolute change in efficiency to be less than $0.01 \%$; spacing increments are $3 \mu \mathrm{m}$. Eight to ten iterations are sufficient to achieve convergence. Section 3.1 below describes the optimization process for an ideal uniform irradiance distribution; Section 3.2 describes the process for a nonuniform irradiance distribution.

3.1. Under Uniform Illumination. Figure 4 shows efficiency as a function of finger spacing at concentrations of 500, 1000, 2000, and 2500 suns under a uniform illumination profile $\left(1\right.$ sun $\left.=900 \mathrm{~W} / \mathrm{m}^{2}\right)$. The relationship between the shortcircuit current and the concentration is assumed to be linear [2]. This implies that the short-circuit current for this cell at 1000 suns is 1000 times that of the short-circuit current under 


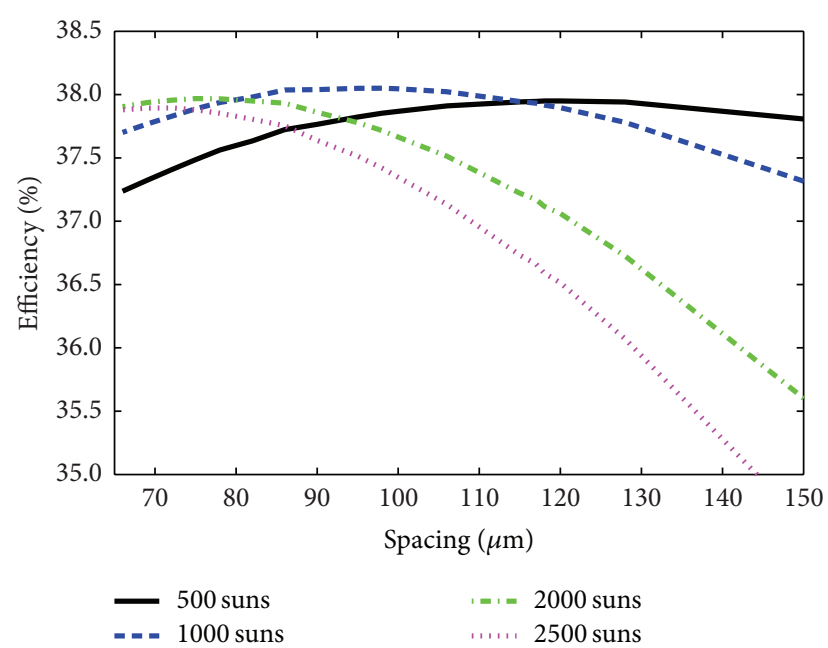

FIGURE 4: Efficiency as a function of spacing at 500, 1000, 2000, and 2500 suns under uniform illumination. An optimal finger spacing is obtained using a Golden section search algorithm after 8-10 iterations.

one sun operation. The figure reveals that the efficiency of the cell reaches a maximum value at each concentration for a specific finger spacing.

The optimal finger spacing at 500 suns is found to be $120 \mu \mathrm{m}$. However, at 1000 suns the optimal finger spacing is reduced to $95 \mu \mathrm{m}$. It is evident that this cell can perform almost optimally, without much loss in efficiency $(<0.2 \%)$, at both 500 and 1000 suns if the fingers are spaced from 95 to $120 \mu \mathrm{m}$. At 2000 and 2500 suns, however, the cell needs to be designed with more closely spaced fingers (optimal spacing is $75 \mu \mathrm{m}$ and $72 \mu \mathrm{m}$ resp.) in order to get the best possible efficiency. A similar trend is reported in an NREL technical report [19]. The highest efficiencies obtained with this cell under uniform illumination are $37.9 \%$ at 500 suns, $38.1 \%$ at 1000 suns, $38.0 \%$ at 2000 suns, and $37.9 \%$ at 2500 suns.

An increase in the slope of the efficiency versus spacing curve with concentration indicates that the penalty for using a nonoptimal spacing is greater at higher concentrations (2000 and 2500 suns) than at lower concentrations (500 and 1000 suns). If the cell is designed with a $120 \mu \mathrm{m}$ spacing and operated under uniform illumination at a concentration of 2500 suns, a $1.3 \%$ absolute loss in efficiency is observed as compared to that obtained with the optimal spacing.

\subsection{Uniform Spacing under Nonuniform Illumination. To} model nonuniform illumination, three Gaussian profiles with different peak to average ratios (PARs) are used to represent various types of concentrating optics. Efficiency optimization is performed for PARs of 1.6, 2.6, and 5.3 at concentrations of 500, 1000, 2000, and 2500 suns across the range of finger spacings. A PAR of 1.6 approximates an ideal concentrating system, while a PAR of 5.3 may represent a system with only a primary Fresnel lens element and no secondary homogenizer. Note that, while the total intensity of each irradiance profile is kept constant, chromatic aberration effects are ignored in the optimization. This implies that current mismatch between top, middle, and bottom subcells is neglected. Figure 5 shows how the efficiency variation with finger spacing depends on the illumination profile; uniform illumination has also been included, for comparison. The three Gaussian irradiance distributions are shown in the inset. The optimal finger spacing is seen to decrease with both increasing nonuniformity and increasing concentration, and the efficiency is likewise reduced. Figure 5(a), at 500 suns, shows that, for the highest PAR, the efficiency at the optimal uniform spacing of $120 \mu \mathrm{m}$ is about $0.5 \%$ absolute lower than it would be at its optimal $90 \mu \mathrm{m}$ spacing; for Figure 5(b), at 1000 suns, the efficiency at the optimal uniform spacing of $98 \mu \mathrm{m}$ is about $0.9 \%$ absolute lower than it would be at its optimal $69 \mu \mathrm{m}$ spacing; and for Figure 5(c), at 2000 suns, the efficiency at the optimal uniform spacing of $75 \mu \mathrm{m}$ is about $1.5 \%$ absolute lower than it would be at its optimal $50 \mu \mathrm{m}$. The results clearly illustrate that an increase in concentration and in nonuniformity has an increasingly significant effect on the conversion efficiency of the solar cell.

\subsection{Nonuniform Finger Spacing under Nonuniform Illumina-} tion. In order to further improve the efficiency of the cell exposed to a nonuniform irradiance profile, a nonuniform grid spacing is investigated to ascertain if unevenly spaced fingers enhance cell efficiency. To reduce computational complexity, the cell is virtually divided into a number of segments of equal area. Spacing is uniform within a segment but differs between segments. For example, consider a solar cell virtually divided into ten segments (say $S_{1}$ to $S_{10}$ ). Since the segments closest to the center of the cell will receive higher illumination, the fingers should be more narrowly spaced at the center of the cell than at the edges. Also, since Gaussian irradiance profiles have been used to represent nonuniform illumination, a symmetric spacing distribution can be used. For example, the spacing for the two outermost segments, $S_{1}$ and $S_{10}$, is the same.

Simulations show a negligible change in efficiency $(<0.01 \%)$ when the number of segments is increased beyond ten. Therefore, ten segments are used for our model. Each segment consists of a number of functional blocks given by

$$
Y=m * \operatorname{Seg} N+C,
$$

where $\operatorname{Seg} N$ is the segment number for the symmetric left half of the cell, $m$ is the slope of the linear profile, and $C$ is a constant.

By varying the values of $m$ and $C$, the number of functional blocks, and hence the finger spacing, is varied. Changing $m$ varies the spacing between segments more drastically than changing $C$. In order to implement this in SPICE, recalculation of resistance values for each segment, and for intermediate areas between segment transitions, is required. Maximum slope $(\operatorname{Max} M)$ and constant $(\operatorname{Max} C)$ values are set to 10 and maximum efficiencies are obtained as the values of $m$ and $C$ are varied between 1 and 10, yielding optimal configurations for all irradiance profiles and concentrations. Figure 6 shows results for a Gaussian irradiance profile of $\mathrm{PAR}=5.3$ at 1000 suns and 2500 suns. At 1000 suns, a maximum efficiency $\left(\eta_{\max }\right)$ of $36.9 \%$ is obtained at $m=2$ and 


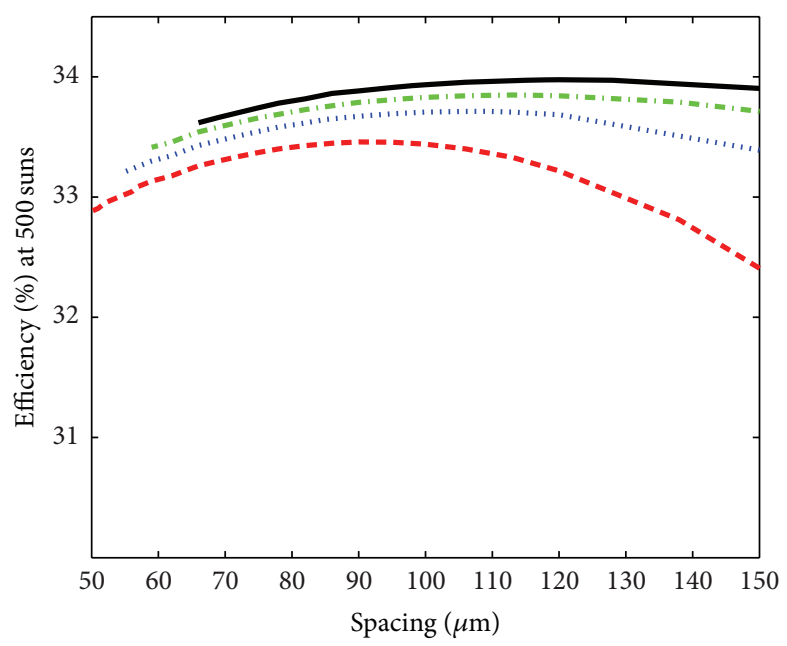

(a)

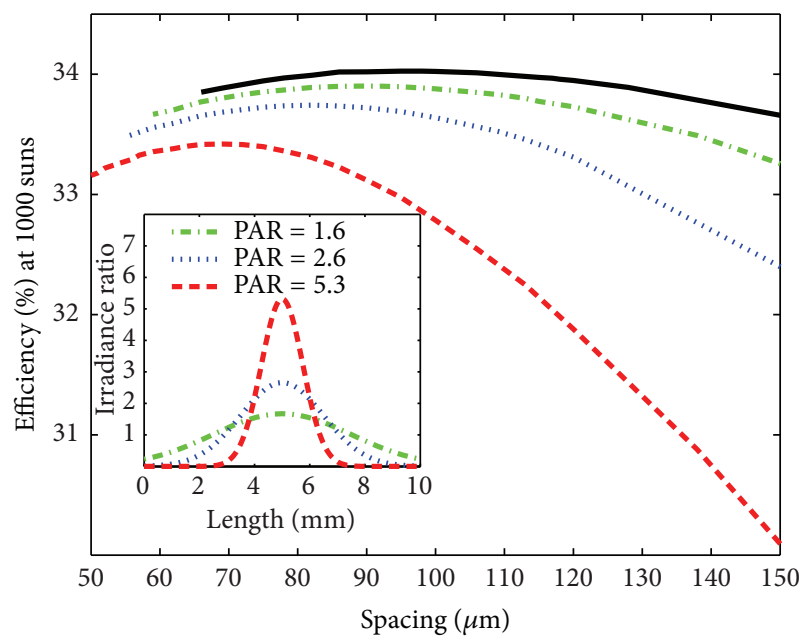

(b)

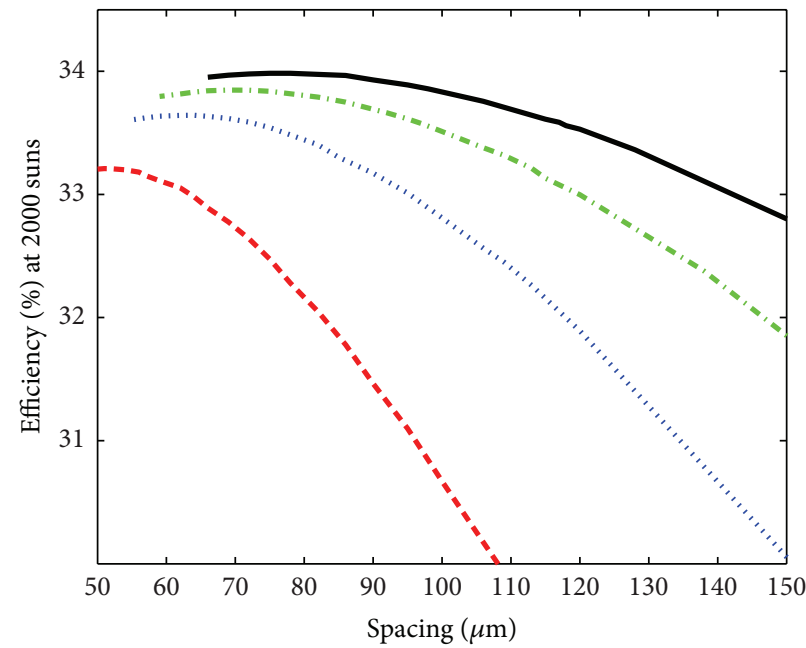

- Uniform illumination

$\ldots$.... Gaussian with PAR $=2.6$

$1=:=$ Gaussian with PAR $=1.6$

- - - Gaussian with PAR = 5.3

(c)

Figure 5: Efficiency as a function of finger spacing at a concentration of (a) 500 suns, (b) 1000 suns, and (c) 2000 suns; inset: Gaussian irradiance distributions with different peak to average ratios (PARs).

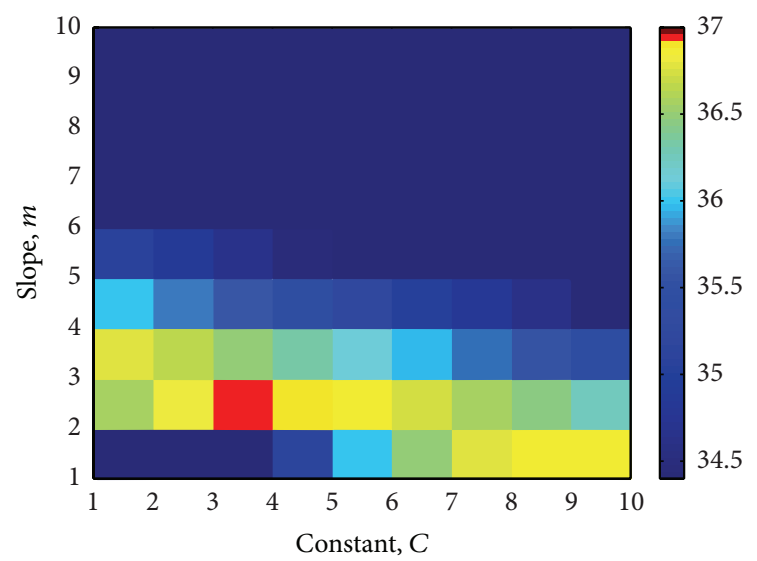

(a)

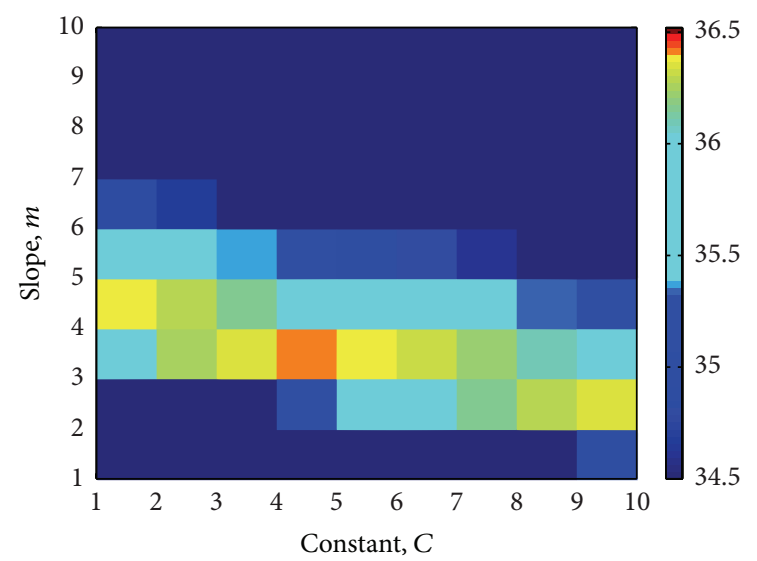

(b)

FIGURE 6: Efficiency as a function of slope $m$ and constant $C$ under a Gaussian irradiance distribution (PAR = 5.3) at (a) 1000 and (b) 2500 suns. The color bar indicates the efficiency values (\%) obtained with each combination. 
TABLE 2: Maximum efficiency $\left(\eta_{\max }\right)$ comparison with uniform and nonuniform spacing under PAR $=5.3$ and PAR $=2.6$ at various concentrations $(X)$.

\begin{tabular}{|c|c|c|c|c|}
\hline \multirow[b]{2}{*}{$X$} & \multicolumn{2}{|c|}{$\mathrm{PAR}=5.3$} & \multicolumn{2}{|c|}{$\mathrm{PAR}=2.6$} \\
\hline & $\begin{array}{c}\eta_{\max } \\
\text { Uniform spacing [\%] }\end{array}$ & $\begin{array}{c}\eta_{\max } \\
\text { Nonuniform spacing [\%] }\end{array}$ & $\begin{array}{c}\eta_{\max } \\
\text { Uniform spacing [\%] }\end{array}$ & $\begin{array}{c}\eta_{\max } \\
\text { Nonuniform spacing [\%] }\end{array}$ \\
\hline 500 & 36.9 & 37.0 & 37.4 & 37.4 \\
\hline 1000 & 36.8 & 36.9 & 37.5 & 37.5 \\
\hline 2000 & 36.4 & 36.6 & 37.3 & 37.4 \\
\hline 2500 & 36.1 & 36.4 & 37.1 & 37.2 \\
\hline
\end{tabular}

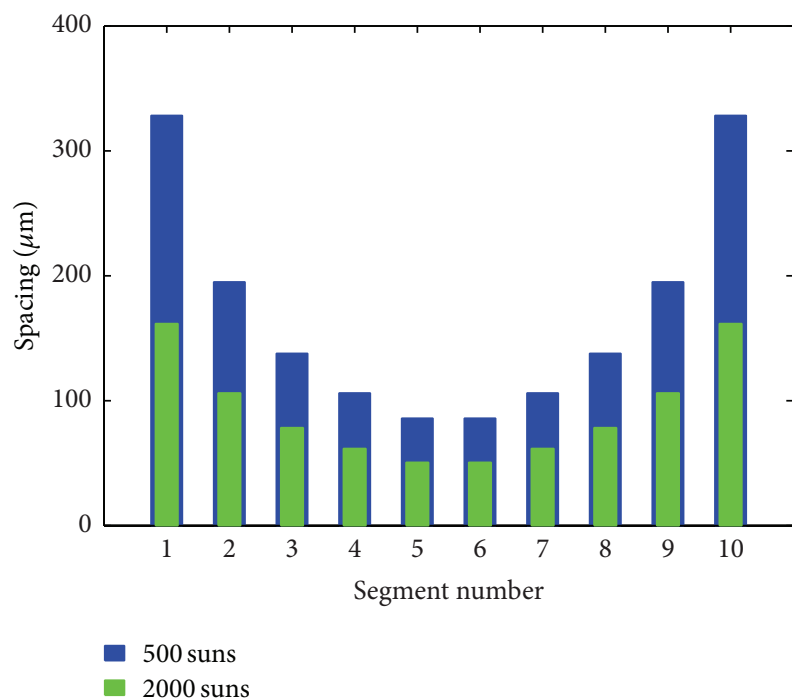

FIGURE 7: Optimal nonuniform spacing profile at (a) 500 suns and (b) 2000 suns under a Gaussian irradiance profile $(\mathrm{PAR}=5.3)$ with the solar cell divided into ten equal segments.

$C=3$. In this case the spacing varies from $195 \mu \mathrm{m}$ at the cell edges to $72 \mu \mathrm{m}$ at the cell center. At 2500 suns, $\eta_{\max }=36.4 \%$ is obtained at $m=3$ and $C=4$. In this case the spacing varies from $137 \mu \mathrm{m}$ at the cell edges to $48 \mu \mathrm{m}$ at the cell center.

Figure 7 shows the optimal spacing profile as a function of segment position under nonuniform irradiance profile with a $\mathrm{PAR}=5.3$ at concentrations of 500 and 2000 suns. It is evident that the spacings become narrower toward the center of the cell. For 500 suns, the spacing varies from $328 \mu \mathrm{m}$ at the cell edges to $86 \mu \mathrm{m}$ at the cell center; and for 2000 suns, it varies from $162 \mu \mathrm{m}$ at the cell edges to $51 \mu \mathrm{m}$ at the cell center.

Similar simulations have been carried out for illumination profiles with PAR $=1.6$ and PAR $=2.6$. Negligible change in maximum efficiency was seen for the near-optimal profile $(\mathrm{PAR}=1.6)$. Table 2 shows the maximum efficiency for Gaussian profiles having a PAR of 2.6 and 5.3 at various concentrations.

For lower concentrations and lower PAR values, the penalty for using uniform spacing is minimal. A similar result has been reported in [20] at 1000 suns. However, at higher concentrations, a $0.3 \%$ absolute efficiency gain is observed. The effect of using a nonuniform spacing is more pronounced as the irradiance PAR increases.

\section{Conclusion}

Our results indicate that, under uniform illumination, the finger spacing needs to be optimized for the system concentration. Under nonuniform illumination, the effect of finger spacing on solar cell efficiency can be pronounced. For uniform spacing at 2500 suns, an efficiency enhancement of $2 \%$ absolute is obtained when optimizing using a Gaussian profile of PAR = 5.3 rather than a flat profile. For nonuniform spacing an additional enhancement in absolute efficiency of $0.3 \%$ was found. At lower concentrations, the penalty for using evenly spaced fingers becomes negligible. The need to optimize grid designs based on nonuniform illumination is thus clearly demonstrated by our optimization of front contact finger spacing on multijunction solar cells using more realistic irradiance profiles across a range of concentrations.

\section{Conflict of Interests}

The authors declare that there is no conflict of interests regarding the publication of this paper.

\section{Acknowledgments}

The authors would like to thank the National Science and Engineering Research Council of Canada, the Canadian Foundation for Innovation, Canada Research Chair Program, Ontario Research Fund, and Cyrium Technologies Inc. for supporting this work and CMC Microsystems for providing access to Sentaurus software.

\section{References}

[1] F. Dimroth, M. Grave, P. Beutel et al., "Wafer bonded fourjunction GaInP/GaAs//GaInAsP/GaInAs concentrator solar cells with $44.7 \%$ efficiency," Progress in Photovoltaics: Research and Applications, vol. 22, pp. 277-282, 2014.

[2] C. Algora, "The importance of the very high concentration in third-generation solar cells," in Next Generation Photovoltaics, pp. 108-136, Institute of Physics Publishing, London, UK, 2003.

[3] L. Fu, R. Leutz, and H. P. Annen, "Secondary optics for Fresnel lens solar concentrators," in 7th Nonimaging Optics: Efficient Design for Illumination and Solar Concentration, vol. 7785 of Proceedings of SPIE, San Diego, Calif, USA, August 2010.

[4] R. Herrero, M. Victoria, C. Domínguez, S. Askins, I. Antón, and G. Sala, "Concentration photovoltaic optical system irradiance distribution measurements and its effect on multi-junction 
solar cells," Progress in Photovoltaics: Research and Applications, vol. 20, no. 4, pp. 423-430, 2012.

[5] C. Algora and V. Diaz, "Influence of series resistance on guidelines for manufacture of concentrator p-on-n GaAs solar cells," Progress in Photovoltaics: Research and Applications, vol. 8, pp. 211-225, 2000.

[6] H. Baig, K. C. Heasman, and T. K. Mallick, "Non-uniform illumination in concentrating solar cells," Renewable and Sustainable Energy Reviews, vol. 16, no. 8, pp. 5890-5909, 2012.

[7] H. Baig, N. Sarmah, K. C. Heasman, and T. K. Mallick, "Numerical modelling and experimental validation of a low concentrating photovoltaic system," Solar Energy Materials and Solar Cells, vol. 113, pp. 201-219, 2013.

[8] J. Nelson, The Physics of Solar Cells, Imperial College Press, London, UK, 2003.

[9] A. W. Walker, Bandgap engineering of multi-junction solar cells using nanostructures for enhanced performance under concentration [Ph.D. dissertation], Dept. of Physics, University of Ottawa, Ottawa, Canada, 2013.

[10] A. W. Walker, O. Thériault, M. M. Wilkins, J. F. Wheeldon, and K. Hinzer, "Tunnel-junction-limited multijunction solar cell performance over concentration," IEEE Journal of Selected Topics in Quantum Electronics, vol. 19, no. 5, pp. 1-8, 2013.

[11] J. F. Wheeldon, C. E. Valdivia, A. W. Walker et al., "Performance comparison of AlGaAs, GaAs and InGaP tunnel junctions for concentrated multijunction solar cells," Progress in Photovoltaics: Research and Applications, vol. 19, no. 4, pp. 442-452, 2011.

[12] G. Segev, G. Mittelman, and A. Kribus, "Equivalent circuit models for triple-junction concentrator solar cells," Solar Energy Materials and Solar Cells, vol. 98, pp. 57-65, 2012.

[13] P. Sharma, A. Walker, J. Wheeldon, H. Schriemer, and K. Hinzer, "Optimization of finger spacing for concentrator photovoltaic cells under non-uniform illumination using SPICE," in Photonics North Conference, vol. 8915 of Proceedings of SPIE, Ottawa, Canada, June 2013.

[14] K. Nishioka, T. Takamoto, T. Agui, M. Kaneiwa, Y. Uraoka, and T. Fuyuki, "Evaluation of InGaP/InGaAs/Ge triple-junction solar cell under concentrated light by simulation program with integrated circuit emphasis," Japanese Journal of Applied Physics, vol. 43, no. 3, part 1, pp. 882-889, 2004, 10.1143/JJAP.43.882.

[15] A. Zekry and A. Y. Al-Mazroo, "A distributed SPICE-model of a solar cell," IEEE Transactions on Electron Devices, vol. 43, no. 5, pp. 691-700, 1996.

[16] B. Galiana, C. Algora, and I. Rey-Stolle, "Comparison of 1D and 3D analysis of the front contact influence on GaAs concentrator solar cell performance," Solar Energy Materials and Solar Cells, vol. 90, no. 16, pp. 2589-2604, 2006.

[17] P. Rodrigo, E. F. Fernández, F. Almonacid, and P. J. PérezHigueras, "Models for the electrical characterization of high concentration photovoltaic cells and modules: a review," Renewable and Sustainable Energy Reviews, vol. 26, pp. 752-760, 2013.

[18] K. Nishioka, T. Takamoto, T. Agui, M. Kaneiwa, Y. Uraoka, and T. Fuyuki, "Evaluation of InGaP/InGaAs/Ge triple-junction solar cell and optimization of solar cell's structure focusing on series resistance for high-efficiency concentrator photovoltaic systems," Solar Energy Materials and Solar Cells, vol. 90, no. 9, pp. 1308-1321, 2006.

[19] R. King, "Ultra-high efficiency multijunction cell and receiver module, Phase 1B: high performance PV exploring and accelerating ultimate pathways," Tech. Rep. NREL/SR-520-47602,
National Renewable Energy Laboratory, Boulder, Colo, USA, 2010.

[20] I. Garcia, C. Algora, I. Rey-Stolle, and B. Galiana, "Study of nonuniform light profiles on high concentration III-V solar cells using quasi-3D distributed models," in Proceedings of the $33 \mathrm{rd}$ IEEE Photovoltaic Specialists Conference (PVSC '08), pp. 1-6, San Diego, Calif, USA, May 2008. 

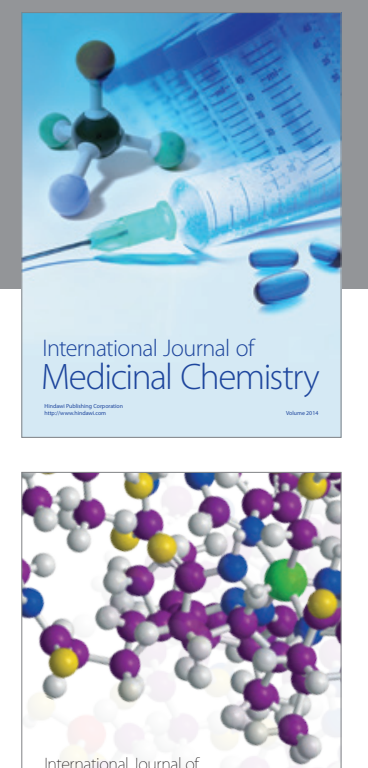

\section{Carbohydrate} Chemistry

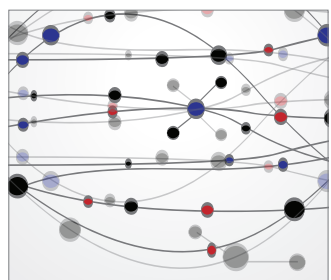

The Scientific World Journal
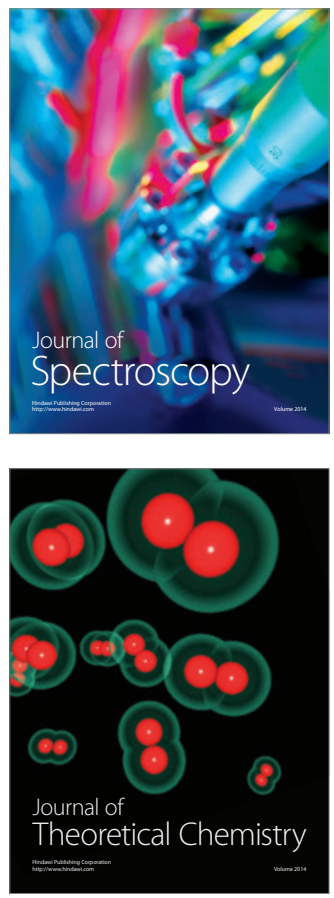
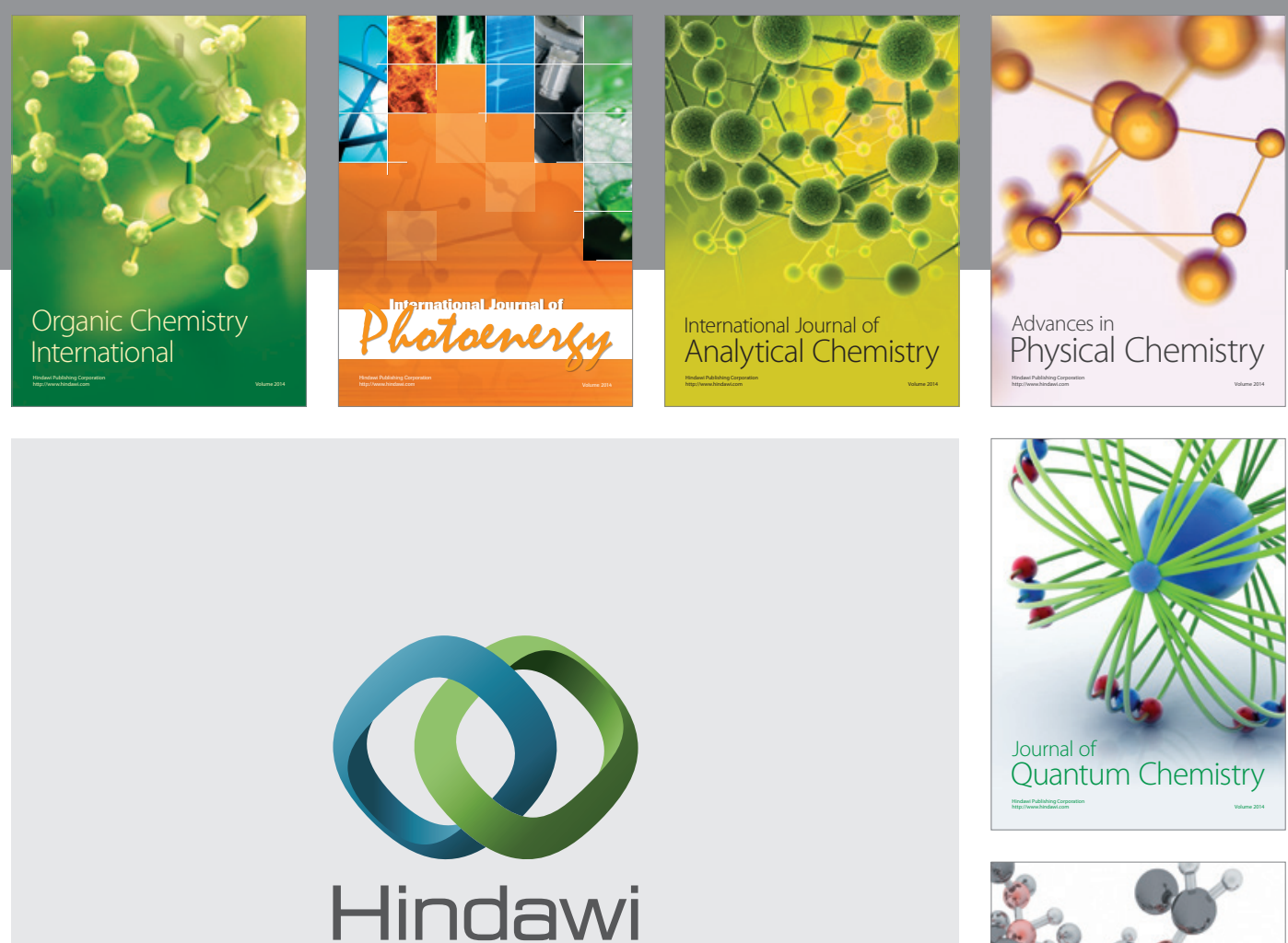

Submit your manuscripts at

http://www.hindawi.com

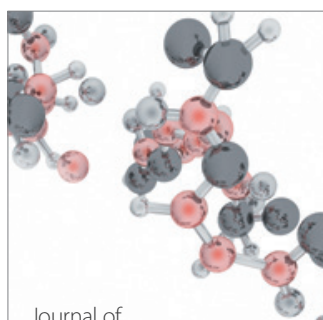

Analytical Methods

in Chemistry

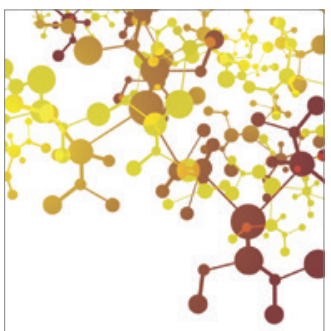

Journal of

Applied Chemistry

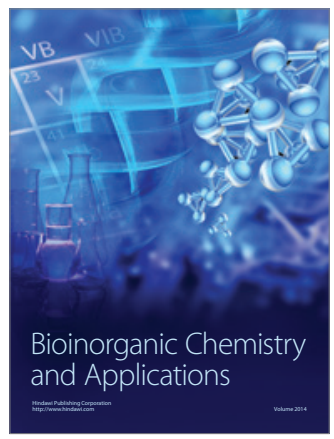

Inorganic Chemistry
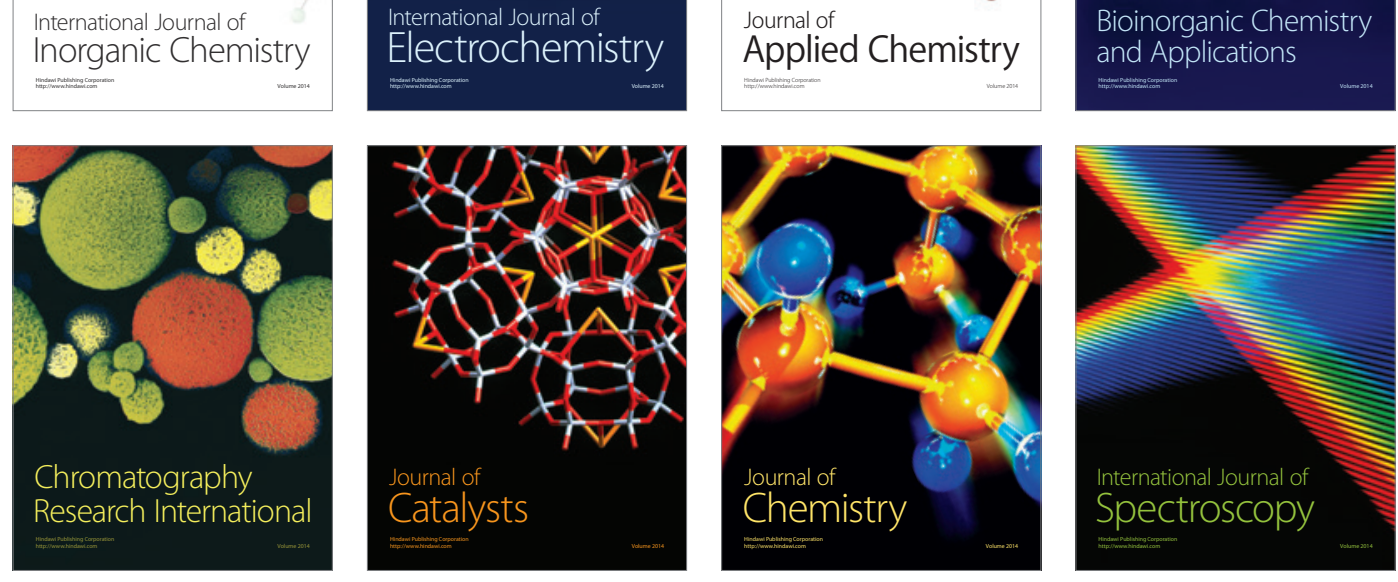